buffer $\mathrm{pH} 4.2$ add $4.5 \mathrm{mg} \mathrm{Na}$-alginate and heat until the alginate is dissolved. After cooling to room temperature add $50 \mathrm{mg}$ o-tolidine $2 \mathrm{HCl}$.

Standard curve for $\mathrm{Hgb}$ : lyze freshly obtained erythrocytes with destilled water, centrifuge and determine Hgb-concentration in the supernatant with the cyanide method (1). Prepare dilutions from $1-10 \mu \mathrm{g} \mathrm{Hgb} / \mathrm{m} /$ with saline and measure the peroxidase activity with the standard method. A straight line should be obtained by plotting max. absorbance over the amount of Hgb (compare fig. 6a).

Standard curve for $\mathrm{Hgb}$-Hp in the presence of $\mathrm{Na}$-alginate: Prepare Hpsolution with $8 \mu \mathrm{g}$ pure $\mathrm{Hp} / 1 \mathrm{ml}$ dest. water (the actual amount of the Hp-preparation depends on its purity). Mix equal volumes of the $\mathrm{Hp}$-solution and a standardized Hgb-solution, containing $8 \mu \mathrm{g}$ $\mathrm{Hgb} / \mathrm{ml} .1 \mathrm{ml}$ of the mixture contains $4 \mu \mathrm{g} \mathrm{Hgb}$ as Hgb-Hp-complex. Prepare dilutions $0.5-4 \mu \mathrm{g} \mathrm{Hgb} / \mathrm{ml}$ and measure the peroxidase activity as described, but substitute the plain o-tolidinetartrate-buffer with the alginate containing solution. In plotting max. absorbance over the amount of Hgb a curve as shown in figure $6 \mathrm{~b}$ will be obtained.

Unknown urine: Dilute urine 1:500 with saline and use $1 \mathrm{ml}$ in the standard method. If the max. absorbance is at least .1, read Hgbconcentration from standard curve and multiply by 500 . If the
Hgb-concentration in the urine is too low to permit a 1:500 solution (no measurable reaction), the urine has to be treated with sephadex.

Prepare a Sephadex-G-50 column $(0.8 \times 20 \mathrm{~cm})$ with $1 \%$ saline. To $0.5 \mathrm{ml}$ urine add $2.5 \mathrm{mg} \mathrm{Hp}$ (calculated as pure $\mathrm{Hp}$ ) and mix well. Put the $5.0 \mathrm{ml}$ on the Sephadex column, elute with $1 \%$ saline and collect $2.0 \mathrm{ml}$ fractions (approx. 10). Take an appropriate aliquot (up to $1.0 \mathrm{ml}$ ) of each fraction and measure the peroxidase activity using the reagent-buffer with Na-alginate. With aliquots less than $1.0 \mathrm{~m} l$ make up the volume difference with saline. For each fraction the amount of $\mathrm{Hgb}$ per $2.0 \mathrm{ml}$ fraction is calculated (standard curve c). The sum is the amount of $\mathrm{Hgb}$ per $5.0 \mathrm{ml}$ of urine.

For each Sephadex column the percentage of recovery of Hgb-peroxidase is determined. Prepare Hgb-solution with $4 \mu \mathrm{g} \mathrm{Hgb} / \mathrm{ml}$ saline and add an equal volume of a Hp-solution with $4 \mu \mathrm{g}$ pure $\mathrm{Hp} / \mathrm{m} l$. From this mixture put $5.0 \mathrm{ml}$ (=10 $\mu \mathrm{g} \mathrm{Hgb}$ as Hp-complex) on column and procede as described for urine. The percent recovery obtained from this determination is used to correct the value obtained for urines with this particular column. The columns can be washed with saline and used repeatedly.

The authors wish to thank Mrs. Henn-DeBruin and Miss Erica ENZER for skillfull technical assistance.

\title{
References
}

1. Hainline, A., Std. Methods Clin. Chem. 2, 49 (1958). 2. Flick, F. W. and C. J. WAtson, J. biol. Chemistry 146, 171 (1942). - 3. Crosby, W. H. and F. W. Furth, Blood 11, 380 (1956). - 4. Mattenhermer, H., and E. C. Adams, Abstracts, 143rd Meeting Amer. Chem. Soc., p. 42 A (1963). - 5. BeAU,
A. F., Amer. J. Clin. Path. 38, 111 (1962). - 6. Lewrs, G. P., J. Clin. Path. 18, 235 (1965). - 7. Conneli, G. E., and O. SMITHIEs, Biochem. J. 72, 115 (1959). - 8. Lupovitch, A., and B. ZACK, Clin. chim. Acta (Amsterdam) 9, 49 (1964).

Professor Dr. H. Mattenheimer, M. D. 1753 - West Congress Parkway Chicago, Ill. 60612 / USA

\section{Über die Ausscheidung der Koproporphyrin-Isomere I und III bei Erkrankungen der Leber und des Blutes}

\author{
Von R. CLOtTen und A. DoyeN ${ }^{1}$ ) \\ Aus der Medizinischen Universitätsklinik Freiburg/Brg. (Direktor: Prof. Dr. Dr. h. c. L. Heilmeyer)
}

(Eingegangen am 9. August 1965)

Mit extraktiven, hochspannungselektrophoretischen und papierchromatographischen Methoden wurde die Koproporphyrinausscheidung bei Gesunden, Leberkranken verschiedener Genese und einer Reihe von Bluterkrankungen untersucht und die Bestimmung der Isomeren I und III durchgeführt. Bei den Normalpersonen fanden sich 42-181 (88,6 $\pm 30,2) \mu \mathrm{g} / 24$ Stdn. (Männer) bzw. 43-135 (70,8 $\pm 28,1)$ $\mu \mathrm{g} / 24 \mathrm{Stdn}$. Gesamtkoproporphyrin im Urin. Koproporphyrin III überwiegt bei gesunden Männern und Frauen (64,8 bzw. 62,2\%). Bei Lebererkrankungen zeigen die nichtalkoholischen Cirrhosen ein Vorherrschen des Isomerentyps I, die Alkoholcirrhosen dagegen eine Koproporphyrin III Vermehrung. Bei den Hepatitiden konnte nur bei einem Teil der Fälle ein Úberwiegen von Kopro I nachgewiesen werden, die Mehrzahl der untersuchten Fälle klinisch abklingender Hepatitis näherten sich hinsichtlich der Isomerenverteilung der Norm. Alle überprüften Anämieformen schieden zum Teil beträchtlich gegenüber der Norm vermehrte Koproporphyrinmengen aus. Die Isomerentrennung läßt bei den sideroachrestischen Anämien, Eisenmangelanämien und Panmyelopathien eine signifikante Vermehrung von Kopro III erkennen, wogegen die hämolytischen Anämien eine eindeutige Vermehrung des Isomerentyps I zeigen.

The excretion of coproporphyrin in normal health, in liver ailments of various origin, and in a series of blood ailments was studied by extraction, high voltage electrophoresis and paper chromatography, and isomers I and III were determined. In normal persons the urinary level was $42-181(88.6 \pm 30.2) \mu \mathrm{g} / 24 \mathrm{hr}$. (males) and $43-135(70.8 \pm 28.1) \mu \mathrm{g} / 24 \mathrm{hr}$. total coproporphyrin. Coproporphyrin III was predominant in healthy men and women ( 64.8 and $62.2 \%$ resp.). In ailments of the liver, non alcoholic cirrhosis was accompanied by a prevelance of isomer I; alcoholic cirrhosis, however, caused an increase of coproporphyrin III. Isomer I only predominated in a few cases of hepatitis; most of the cases of clinical hepatitis showed a distribution of isomers similar to the normal. All cases of anaemia excreted considerably increased amounts of coproporphyrin. In sideroachrestic anaemia, iron deficiency anaemia and panmyelopathies, there was a significant increase of isomer III, while haemolytic anaemias showed a marked increase of isomer I.

Die Bestimmung der mit dem Harn, der Galle oder dem Stuhl ausgeschiedenen Porphyrine ist für die Diagnose

1) Diese Arbeit wurde mit Unterstützung der Deutschen Forschungsgemeinschaft durchgeführt. und Klassifizierung der verschiedenen Formen der idiopathischen oder erworbenen Porphyrien von entscheidender Bedeutung. Diese Nebenprodukte der Haemsynthese werden vor allem in der Leber und dem 
blutbildenden Mark durch in vivo wahrscheinlich irreversible Oxydation der entsprechenden reduzierten Porphyrinogene gebildet. Sie stellen eine in dieser Form nicht mehr verwertbare, physiologisch funktionslose Gruppe von Tetrapyrrolen dar, die, da sie im Gegensatz zu den Gallenfarbstoffen auch nicht weiter abgebaut werden können, der Ausscheidung auf dem einen oder anderen Weg anheimfallen. Dabei werden von den bisher nachgewiesenen natürlicherweise vorkommenden Porphyrinen mit zwei bis acht Carboxylgruppen die niedrig carboxylierten Vertreter ausschließlich (Di- und Tricarboxylporphyrine) oder überwiegend (Koproporphyrine) über die Galle mit dem Stuhl ausgeschieden, während die entsprechenden Verbindungen mit mehr als 4 Carboxylgruppen zum größten Teil im Urin erscheinen.

Die Bestimmung der täglich so eliminierten Porphyrine und ihrer reduzierten Vorstufen sowie die Erfassung der bisher ausschließlich nachgewiesenen Isomere der Reihe I und III (für ein natürliches Auftreten der Isomere II und IV gibt es bis jetzt keinen überzeugenden Anhalt), macht bei den zum Teil mit enormen Porphyrinausscheidungen einhergehenden hepatischen und erythropoetischen Porphyrien keine Schwierigkeiten. Anders liegen die Verhältnisse jedoch bei der Erfassung dieser Stoffe in den Ausscheidungen Gesunder oder bei solchen Erkrankungen, die nur mit einer mäßigen Vermehrung von Porphyrinen verbunden sind. Hier liegen, zum größten Teil aus methodischen Gründen, teilweise sehr widerspruchsvolle Angaben über Art, Menge und Isomerenverteilung dieser Verbindungen, insbesondere für die bisher vorwiegend untersuchten Kopro- und Uroporphyrine vor (1-9). Vergleicht man die in den älteren Veröffentlichungen berichteten Harn-Koproporphyrinausscheidungen mit neueren Werten, die mit zuverlässiger und empfindlicherer Methodik erhalten wurden, so darf heute unter normalen Verhältnissen mit einer Koproporphyrinausscheidung von etwa 40-180 $\mu \mathrm{g} / 24$ Stdn. bei Mittelwerten um $80 \mu \mathrm{g} / 24$ Stdn. zu rechnen sein.

Wesentlich divergentere Auffassungen als über die tatsächlich normalerweise im Urin zu erwartenden Koproporphyrinkonzentrationen bestehen über die dabei zu beobachtende İsomerenverteilung. Während lange Zeit ein Überwiegen des Isomerentyps I angenommen wurde $(1,10-16)$, konnten in den letzten Jahren eindeutige Befunde dafür erhoben werden, $\mathrm{da} B$ auch unter normalen Verhältnissen mindestens $50 \%$ der mit dem Harn ausgeschiedenen Koproporphyrine dem Isomerentyp :III angehören $(3,8,9,17)$. Damit konnten ältere, gleiçhlautende Befunde von Grotepass (18), Brugsch (4) und KEHL (5) bestätigt werden.

Nachdem die Leber neben den erythropoetischen Elementen des Knochenmarks den überwiegenden Anteil der normalerweise vom Organismus benötigten Tetrapyrrolchromoproteide liefert und eine Reihe - vor allem chronischer - Lebererkrankungen mit einer mehr oder weniger vermehrten Ausscheidung von Koptoporphyrin einhergehen, war es naheliegend die qualita- tiven und quantitativen Verhältnisse solcher Porphyrinurien einer näheren Untersuchung zu unterziehen. Erste Untersuchungen dieser Art wurden 1935 von WaTSON (19) durchgeführt, der aus dem Urin eines Patienten mit fortgeschrittener Lebercirrhose Koproporphyrin I isolierte. Dobriner (20) fand bei 22 von 24 Leberkranken (katarrhalischer bzw. Verschlußikterus, Cirrhosen, hämolytischer Ikterus) eine vermehrte Ausscheidung von Koproporphyrin I. VIGLIANI und LiBowritsky (21) untersuchten 3 Gruppen von lebercirrhotischen Patienten und fanden bei einer Gruppe eine isolierte vermehrte Ausscheidung von Kopro-I, bei einer zweiten etwa gleiche Anteile beider Isomeren, während die dritte Gruppe ausschließlich Koproporphyrin III ausschied. Etwa die gleichen heterogenen Ergebnisse wurden von Nesbitr (22) exhalten. Dagegen fanden $W_{A T S O N}$ und Mitarbeiter (1) bei der Untersuchung von 215 Leberkranken (Virushepatitiden, Pankreas- und Gallenwegskarzinom, Steingallen, Lebercirrhosen) von wenigen Ausnahmen abgesehen ein Überwiegen von Koproporphyrin I. Sie vertraten die Auffassung, daß eine vermehrte Ausscheidung des Isomerentyps III für das Vorliegen einer neoplastischen Erkrankung verdächtig sei. 1951 wurden von WaTSON, SUTHERLAND und HAW KINSON (23) 88 weitere Cirrhosekranke hinsichtlich ihrer Koproporphyrinausscheidung und der dabei vorliegenden Isomerenverteilung untersucht, um "etwa vorhandene Unterschiede zwischen alkoholbedingten und nichtalkoholischen Cirrhosen aufzudecken. Dabei fanden sie bei den Alkoholcirrhosen eine sichere Vermehrung des Isomerentyps III, ein Befund, der von KEHL (24) bestätigt werden konnte. Auch bei der Alkoholvergiftung überwiegt die Ausscheidung von Koproporphyrin III (25). Demgegenüber sprechen die neueren Befunde von KeHL (24) sowie von AzIz, SChwartz und Watson (9) bei der Virushepatitis, dem Verschlußikterus, der nichtalkoholischen Cirrhose, der Fettcirrhose und der kleinknotigen Cirrhose für ein Überwiegen von Koproporphyrin I im Urin.

Im Hinblick auf diese Untersuchungen interessierte inwieweit der Koproporphyrinbestimmung und insbesondere dem prozentualen Nachweis der einzelnen Isomere bei Leberkrankheiten eine diagnostische und vor allem prognostische Bedeutung zukommt. Darüber hinaus erschien es interessant zu prüfen, ob andere Erkrankungen, die mit einer absoluten Vermehrung des Harnkoproporphyrins einhergehen, wie $z$. B. bestimmte Blutkrankheiten, hier signifikante und charakteristische Veränderungen zeigen.

\section{Methodik}

Zur Festlegung der Normalausscheidung wurden 40 gesunde Versuchspersonen beiderlei Geschlechts hinsichtlich ihrer Koproporphyrinausscheidung untersucht. Zum Ausschluß latenter Erkrankungen, besonders solcher der Leber, wurden vorher Serumeisen, Transaminasen, Hämoglobin, Erythrocyten, Hämatokrit, Leukocytenzahl und Differentialblutbild untersucht, und eine Serumeiweißelektrophorese durchgeführt. - Die von uns untersuchten pathologischen Fälle umfaßten 12 Virushepatitiden, 1 akute gelbe Leberatrophie, 18 Lebercirrhosen verschicdenen 
Ausmaßes und unterschiedlicher Genese, 12 schwere Eisenmangelanämien, 11 erworbene sideroachrestische Anämien, 4 Panmyelopathien, 4 hämolytische Anämien verschiedener Genese, 1 Cooley Anämie, 2 Leukämien, $1 M$. Hodgkin und 1 schwere Blutungsanämie.

Sämtliche Urine wurden für 24 Stunden streng im Dunkeln und kühl über etwa $5 \mathrm{~g}$ Natriumbikarbonat gesammelt. Danach wurden die Proben entweder sofort aufgearbeitet oder bis zu 48 Stunden bei $+4^{\circ}$ im Kühlschrank gelagert.

Die Extraktion der Koproporphyrine erfolgte nach der von Askevold (26) angegebenen Modifizierung der von SAILLET (27) beschriebenen Methode, bzw., besonders bei stark gefärbten Harnen, hochspannungselektrophoretisch nach vorheriger Adsorption der Porphyrine an Talkum (7).

Die Bestimmung der Erythrocytenporphyrine erfolgte in einer Modifizierung der von ScHWARTz und WIKOFF (28) angegebenen Methode (29).

Die spektrophotometrische Bestimmung wurde nach der von Rimington und Mitarbeiter (30) und von WITH (31) vorgeschlagenen Methode durchgeführt.

Zur Bestimmung der Koproporphyrin-Isomeren wurde die von ERIKSEN (32) angegebene papierchromatographische Methode verwendet. Als Kontrollen dienten chromatographisch reine Koproporphyrin-Isomere I und III.

\section{Ergebnisse}

\section{Normalfälle}

Die mit der beschriebenen Methodik im Harn gesunder Versuchspersonen mittleren Lebensalters gefundenen Koproporphyrinausscheidungen lagen zwischen 42 und $181 \mu \mathrm{g} / 24$ Stdn. (Männer 42-181 $\mu \mathrm{g} / 24$ Stdn., Frauen 43-135 $\mu \mathrm{g} / 24 \mathrm{Stdn}$.) mit einer mittleren Streuung von $88,6 \pm 30,2 \mu \mathrm{g}$ bei Männern und 70,8 $\pm 28,1 \mu \mathrm{g}$ bei Frauen. Die Mehrausscheidung bei den Männern ist signifikant $(P<0,05)$. Sowohl bei gesunden Männern als auch bei gesunden Frauen überwiegt die Ausscheidung von Koproporphyrin III mit im Mittel 64,8 bzw. $62,2 \%$. Es gibt jedoch auch unter den gesunden Versuchspersonen Fälle, bei denen ohne erkennbare anamnestische Ursache eine Predominanz des I-Isomeren nachweisbar ist, die bis zum nahezu völligen Fehlen von Koproporphyrin III reichen kann (Abb. 1).

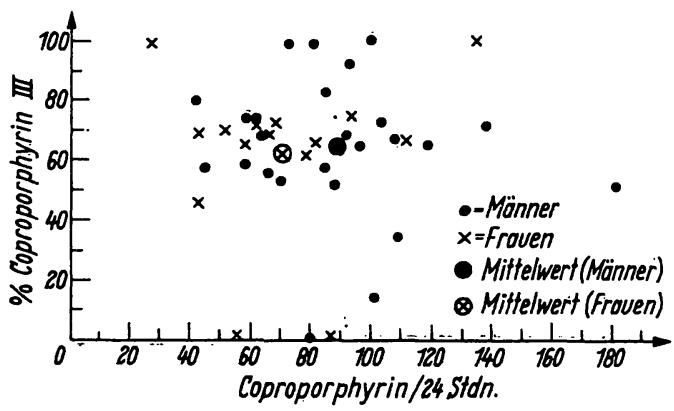

Abb. 1

Verteilung der Koproporphyrinisomere I und III bei Gesunden

\section{Lebererkrankungen}

Zur Untersuchung gelangten 12 Patienten mit einer frischen oder wieder abklingenden Virushepatitis und 18 Patienten mit ausgeprägter teilweise dekompensierter Lebercirrhose verschiedener Ursache. Bei den Hepatitisfällen zeigten nur 2 der 12 Pạtienten eine deutliche über die Norm vermehrte Koproporphyrinausscheidung. Alle anderen Werte liegen im mittleren bis oberèn Normbereich. Möglicherweise ist diese „Normalausscheidung“ darauf zurückzuführen, daß der größere Teil dieser Patienten das floride Stadium der Erkrankung zum Zeitpunkt der Untersuchung bereits hinter sich hatte und sich deshalb das Koproporphyrin, wie auch das Bilirubin und die untersuchten Serumenzyme der Norm näherten (s. Tab. 1).

Tab. 1. Hepatitis-Fälle. Die in Klammern stehenden Werte wurden im Anfạng der Krankheit erhoben.

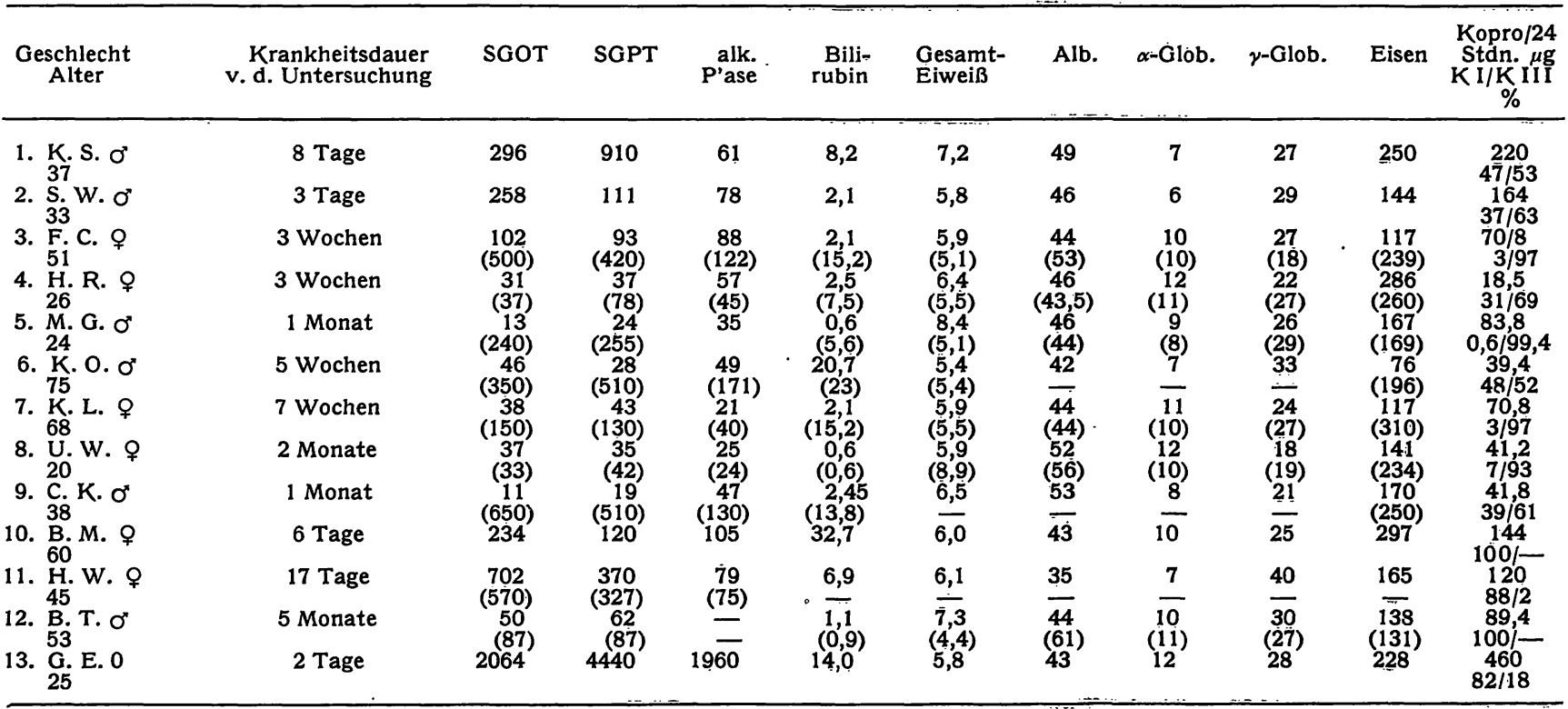

Leberpunktate: 2. S. W. Wenig fortschreitende chronische Hepatitis.

4. H. R.Noch floride chronische Hepatitis.

8. U. W. Subakute bis chronische antikterische Hepatitis.

10. B. M. Schwere lebhaft fortschreitende chronische Hepatitis mit beginnendem cirrhotischen Umbau.

11. H. W. Zweite Hepatitis - fortschreitende ungeordnete Lebercirrhose.

12. B. T. Schwere chronische fortschreitende Hepatitis mit beginnender Lebercirrhose.

13. G. E. Akute Leberzellnekrose. 
Das Ergebnis der Isomerenbestimmung ließ nur bei 4 der Hepatitispatienten eine eindeutige Vermehrung von Koproporphyrin I erkennen, die in einem Fall bis 5 Monate nach dem Beginn der Erkrankung nachweisbar war. Die übrigen Fälle ergaben ein durchschnittliches Verhältnis von etwa $45 \%$ Kopro I zu etwa 55\% Kopro III. Auch hier ist wahrscheinlich die schon weitgehende Normalisierung der Leberfunktion ursächlich für dieses Isomerenverhalten. Bei den frischen Erkrankungsfällen, insbesondere bei der akuten Leberzellnekrose (Fall 13 G. E. Tab. 1) liegt dagegen eine eindeutige Verschiebung zugunsten des Isomerentyps I vor.

Im Vergleich hierzu zeigen die untersuchten Cirrhosefälle (Abb. 2) wenn man von den vorwiegend alkohol-

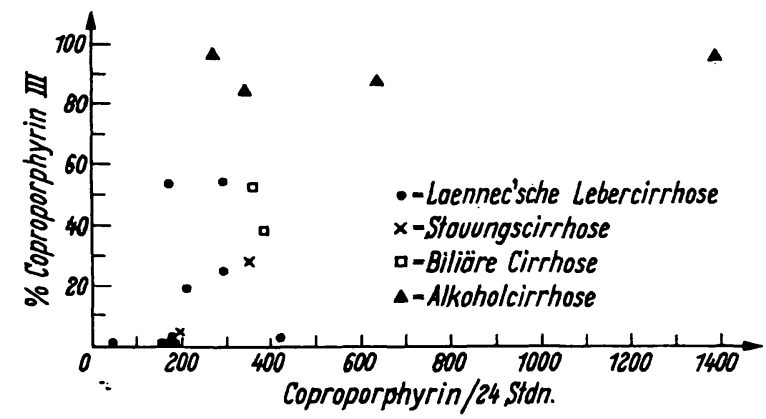

Abb. 2

Verteilung der Koproporphyrinisomere I und III bei Leberkranken. .

bedingten Fällen absieht, ein eindeutiges Überwiegen von Koproporphyrin I, welches im Mittel ungefähr $80 \%$ des Gesamtkoproporphyrins beträgt. Demgegenüber verschiebt sich bei der Alkoholcirrhose das Isomerenverhältnis zugunsten von Koproporphyrin III (83 bis 95\% der Gesamtausscheidung), so daß man bei der Cirrhose das Verhältnis der beiden Isomeren zueinander als differentialdiagnostisches Kriterium heranziehen kann. Auch die Gesamtausscheidung an Koproporphyrin ist bei den Cirrhosefällen höher als bei den akut entzündlichen Lebererkrankungen (im Mittel $335 \mu \mathrm{g} /$ 24 Stdn. gegenüber $121 \mu \mathrm{g} / 24 \mathrm{Stdn}$. bei Hepatitiden). Dabei ist bei den chronischen Alkoholikern eine Tendenz zu höheren Ausscheidungswerten gegenüber der nichtalkoholischen Cirrhose deutlich. Eine Parallelität zwischen der Höhe der Koproporphyrinausscheidung und den sonst erhobenen blutchemischen Leberbefunden ließ sich weder bei der Hepatitis noch bei den verschiedenen Cirrhoseformen nachweișen.

\section{Bluterkerankungen}

Die Ergebnisse det Koproporphyrinbestimmung im Urin bei einer Anzahl anämischer Erkrankungen im Vergleich zu den Kopro- und Protoporphyrinwerten der Erythrocyten dieser Patienten sind in der Abbildung 3 wiedergegeben.

Während bei praktisch allen diesen Erkłankungen eine zum Teil beträchtlich vermehrte Koproporphyrinausscheidung zu beobachten ist, zeigen sich im Verhältnis der Isomeren deutliche und im Vergleich der einzelnen Blutkrankheiten zueinander hoch signifikante Unter-

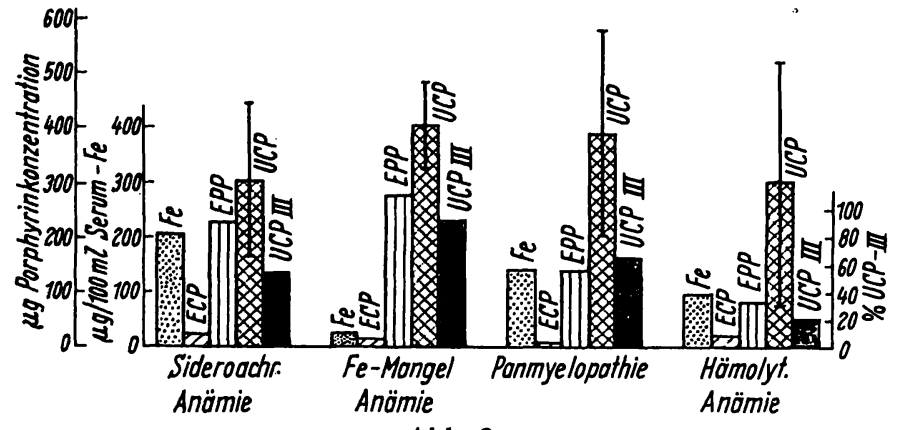

Abb. 3

Vergleich der Koproporphyrinausscheidung (UCP) und des Kopro- und Protoporphyringehaltes der Erythrocyten (ECP bzw. EPP) bei einzelnen Anämieformen.

Der Anteil des bei den verschiedenen Blutkrankheiten nachweisbaren Urinkoproporphyrin III zeigt beim Vergleich der einzelnen Krankheits-

$$
\begin{array}{rl}
\text { gruppen untereinander signifikante Unterschiede } \\
\text { (Gruppe } 1 \text { zu } 2 \text { P }=0,001 & 2 \text { zu } 4 \text { P }=0,001 \\
1 \text { zu } 4 \text { P }=0,001 & 3 \text { zu } 4 \text { P }=0,001 \\
2 \text { zu } 3 \text { P }=0,001 & 1 \text { zu } 3 \text { nicht signifikant }
\end{array}
$$

schiede $^{1}$ ) (s. Legende zu Abb. 3). Die höchsten Koproporphyrinkonzentrationen im Urin werden bei den Eisenmangelanämien gefunden (im Mittel $404 \pm 41 \mu \mathrm{g}$ / 24 Stdn.), Werte, wie sie, wenn auch mit relativ großen Schwankungen, nur noch bei den Panmyelopathien zu beobachten sind ( $388 \pm 172 \mu \mathrm{g} / 24$ Stdn.). Die Koproporphyrinausscheidungen bei den hier untersuchten erworbenen sideroachrestischen Anämien und den hämolytischen Anämien zeigen in etwa das gleiche Bild (308 \pm 141 bzw. $300 \pm 223 \mu \mathrm{g} / 24 \mathrm{Stdn}$.). Während bei den sideroachrestischen Anämien Koproporphyrin I und III etwa zu gleichen Mengen ausgeschieden werden, überwiegt bei den Eisenmangelanämien und den Panmyelopathien deutlich das Isomere III (91 bzw. 66\% des Gesamtkoproporphyrins). Demgegenüber zeigen die hämolytischen Anämien in allen Fällen ein Vorherrschen von Koproporphyrin I (im Mittel $80 \%$ des GesamtKoproporphyrins). Die wenigen untersuchten Fälle weiterer Bluterkrankungen lassen keine eindeutigen Schlüsse auf das Úberwiegen des einen oder anderen Isomerentyps zu. Die bei diesen Erkrankungen gefundenen Werte waren folgende:

\begin{tabular}{lrrrrr}
\hline & ECP & EPP & \multicolumn{2}{c}{ UCP } & \multicolumn{2}{c}{ Isomerentyp (\%) } \\
& \multicolumn{1}{c}{$\mu \mathrm{g} / 100 \mathrm{ml}$} & $\mu \mathrm{g} / 24$ Stdn. & I & III \\
\hline Cooley Anämie & 8 & 76 & 384 & 17 & 83 \\
Morb. Hodgkin & 4 & 82 & 227 & 65 & 35 \\
Leukose, unreifzellig & 6 & 98 & 79 & 42 & 58 \\
Leukämie, chron. myel. & 5 & 63 & 96 & 61 & 39 \\
Blutungsanämie & 5 & 304 & 54 & 12 & 88 \\
\hline
\end{tabular}

Um hier evtl. vorhandene Unterschiede in der Isomerenverteilung festlegen $z u$ können, müßten größere Kontingente solcher Krankheiten untersucht werden, was bisher nicht möglich war.

\section{Diskussion}

Die hier durchgeführten Untersuchungen über die Ausscheidung der Koproporphyrin-Isomeren I und III beim Gesunden stehen in Úbereinstimmung mit neueren

i) Wir sind dem Institut f. Med. Statistik der Univ. Freiburg, Herrn Dr. JESDINSKY für die durchgeführten statistischen Auswertungen sehr zu Dank verpflichtet. 
Untersuchungen anderer Autoren $(8,9,24)$ und bestätigen damit die schon von Grotepass (18) und anderen $(3,17)$ erhobenen Befunde, die für eine zumindest gleichmäßige Verteilung beider Isomeren beim Gesunden sprachen. Wie WATSON und Mitarbeiter (9) konnten auch wir bei den Normalpersonen eine niedrigere Gesamtkoproporphyrinausscheidung bei Frauen als bei Männern feststellen, wobei sich jedoch die Isomerenverhältnisse prozentual nicht verändern. Aus welchem Grunde bei einzelnen Normalpersonen ein Überwiegen von Koproporphyrin I konstant nachweisbar ist, kann vorläufig nicht entschieden werden. Unter Umständen sind solche Ausscheidungen von heute noch nicht abgrenzbaren Faktoren abhängig, wofür auch Untersuchungen von WATSON und Mitarbeitern (1) sprechen würden, die bei einer Gruppe von Gesunden eine Mehrausscheidung von Kopro III fanden, bei einer anderen Gruppe jedoch ein relatives Überwiegen von Koproporphyrin I (48-92\%) beobachten konnten.

Betrachtet man die in der Literatur vorliegenden Angaben über die Höhe der Koproporphyrinausscheidung und die Verteilung der Isomeren bei Leberkrankheiten und die bei unseren Patienten erhobenen Befunde so ergibt sich, daß praktisch alle schwereren Erkrankungen der Leber mit einer zum Teil beträchtlichen Steigerung der Koproporphyrinausscheidung verbunden sind. Mit Ausnahme der Alkoholcirrhose (und Alkoholvergiftung), bei der der Isomerentyp III vorherrscht, findet sich bei allen anderen Cirrhoseformen überwiegend oder ausschließlich Koproporphyrin I (1, 9, 19, 20, 23-25). Nur in einzelnen Fällen konnte bei nichtalkoholischen Cirrhoseformen eine Vermehrung des Isomeren III nachgewiesen werden $(21,22)$. Auch bei der Virushepatitis und beim Verschlußikterus (extra- und intrahepatisch) wird ein Überwiegen von Koproporphyrin I bei meist auch gleichzeitig vermehrtem Koproporphyrin III nachweisbar $(1,4,9,20,24)$. Daß bei unseren Hepatitiden nicht mehr in allen Fällen ein vermehrtes Kopro I nachweisbar war, ist zweifellos darauf zurückzuführen, daß sich bei den meisten dieser Patienten schon eine weitgehende Normalisierung der Ausscheidung eingestellt hatte und die im floriden Stadium nachweisbare Kopro-IVermehrung offenbar rasch wieder verschwindet. Über ähnliche Beobachtungen berichtete auch KeHL (24), die dabei eine relative Vermehrung von Koproporphyrin I bei sonst normalem Koproporphyringehalt für den Ausdruck eines latenten Leberschadens hält. Auf der anderen Seite spricht das Sistieren eines vermehrten I-Isomeren bei gleichzeitig erhöhter Gesamtkoproporphyrinausscheidung nach klinisch abgeklungener Hepatitis mit großer Wahrscheinlichkeit für einen cirrhotischen Umbau der Leber (s. Tab. 1).

Uber die Koproporphyrinausscheidung und die Isomerenverteilung bei Blutkrankheiten liegen bisher kaum Mitteilungen vor $(17,29)$. Bei zahlreichen Anämieformen konnte eine vermehrte Koproporphyrinausscheidung nachgewiesen werden, wobei in den meisten Fällen ein Uberwiegen des. Isomerentyps III zu beobachten war $(29)^{1}$ ). In der Abbildung 3 sind die bei den von uns untersuchten Blutkrankheiten exhobenen Befunde dargestellt, die nur bei. den hämolytischen Anämien konstant eine Vermehrung von Koproporphyrin I erkennen lassen. Nach den Untersuchungen von Dobriner und RHoads (14), Watson und Larson (16) Watson und Mitarbeitern (1) und SchWARTZ und Mitarbeitern (33) lassen sich derartige Kopro-I-Vermehrungen bei allen mit einer gesteigerten erythropoetischen Aktivität einhergehenden Blutkrankheiten nachweisen. Welches nun aber die eigentliche Ursache für die bei einzelnen dieser Erkrankungen zu findende vermehrte Ausscheidung von Koproporphyrin III, bei anderen für die des Isomerentyps I ist, bleibt spekulativ. Es wäre daran zu denken, daß im Falle einer Vermehrung des Typs III, wie sie so ausgesprochen bei den Eisenmangelanämien zu beobachten ist, das für die Hämsynthese zur Verfügung gestellte Koproporphyrinogen gar nicht mehr in Protoporphyrinogen überführt wird (nicht verwertbares Protoporphyrin ist wegen der mangelnden Bereitstellung an Eisen ohnehin in abundanten Mengen vorhanden) und damit nach Oxydation als Schutzmaßnahme des Organismus vor einer Porphyrinüberflutung eliminiert wird.

Anders bei den bämolytischen Anämien und anderen $\mathrm{Zu}$ ständen, die mit einer gesteigerten Erythropoese verlaufen. Hier liegt zweifellos eine gesteigerte Porphyrinbildung zur Kompensierung der Verlustquote vor, bei der der überwiegende Anteil des Koproporphyrinogen III für die Hämbildung verwendet wird, während der nicht verwertbare aber gleichzeitig entstehende Isomerentyp I vermehrt ausgeschieden wird. Daß dies jedoch nicht die einzige Erklärung hierfür ist, geht daraus hervor, daß bei den sideroachrestischen Anämien, die stets von einer gesteigerten Ërythropoese begleitet sind, etwa normale Verhältnisse hinsichtlich der Isomerenverteilung anzutreffen sind - trotz stark vermehrter Gesamtkoproporphyrinausscheidung. Zweifellos spielen hier noch ungeklärte Störungen der Bildung von Hämchromogenen eine unter Umständen entscheidende Rolle.

1) Siehe hier auch zusammenfassende Literaturangaben über entsprechende frühere Untersuchungen.

\section{Literatur}

1. Watson, C. J., V. Hawkinson, S. Schwartz u. D. Sutherrland, J. Clin. Invest. 28, 447 (1949). und Watson, C. J., S. Schwartz, W. Schulze, L. Jacobson und R. ZaGaria, J. Clin. Invest. 28, 465 (1949). - 2. Zieve, L., S. Schwartz und C. J. Watson, J. Lab. u. Clin. Med. 41, 663 (1953). - 3. Comport, A., H. Moore und M. Weatherali, Biochem. J. 58, 117 (1954). - 4. Brugsch, J., Porphyrine, J. A. Barth, 2. Aufl., Leipzig (1959). - 5. KEHL, R.,
Z. klin. Med. 155, I (1958). - 6. BÉNARD, H., A. GajDos und M., GajDos-TöröK, Porphyries, Étude clinique et biologique J. B. Baillière et Fils Ed., Paris (1958). - 7. Cuorren, R. und H. Weringer, Dtsch. med. Wsschr. 87, 131 (1962). - 8. ERIKSEN, L., N. Eriksen und S. HAAvaldDSEN, Scand. J. Clin. Lab. Invest. 14, I (1962). - 9. Aziz, M. A., S. Schwartz und C. J. Watson, J. Lab. Clin. Med. 63, 585 (1964). und AzIz, M. A., S. Schwartz 
und C. J. Watson, J. Lab. Clin. Med. 63, 597 (1964). - 10. HozRBURGER, W., Inaugural Dissertation, Erlangen (1933). - 11. FINK, H. und W. Hoerburger, Naturwiss. 18, 292 (1934). - 12. Watson, C. J., J. Clin. Invest. 15, 327 (1937). - 13. ZeILE, K. und B. RAU, Z. physiol. Chem. 250, 197 (1937). - 14. Dobriner, K. und C. P. Rhoads, Physiol. Rev. 20, 416 (1940). - 15. Schwartz, S., V.HAwkinson, S. Cohen und C. J. Watson, J. Biol. Chem. 168, 133 (1947). -16. Warson, C. J. und L. A. Larson, Physiol. Rev. 27, 478 (1947). -17. Neve, R.A. und R. A. Aldrich, Pediatrics 15, 553 (1955). 18. Grotepass, W., Z. Physiol. Chem. 253, 276 (1938). - 19. Watson, C. J., J. Clin. Invest. 14, 106 (1935). - 20. Dobriner, K., J. Biol. Chem. 113, 1 (1936). - 21. Vigliani, E. C. und H. LibowItskx, Klin. Wschr. 16, 1243 (1937). - 22. NesBirt, S. und A. M. Sneld, Arch. Int. Med. 69, 573 (1942). - 23. Watson, C. J., D. Sutherland und V. Hawkinson, J. Lab. Clin. Med. 37, 8 (1951). -24. KeHL, R., Z. klin. Med. 156, 405 (1960). - 25. SutherLAND, D. und C. J. Watson, J. Lab. Clin. Med. 37, 29 (1951). - 26. Askevold, R., Scand. J. Clin. u. Lab. Invest. 3, 318 (1951). -
27. Sarllet, N. K., Rev. de Med. 16, 542 (1896). (zit. von Watson, Pimenta de Mello, Schwartz, Hawkinson and Bossenmaier 1951). 27a. Watson, C. J., R. Pimenta de Mello, S. Schwartz, V. Hawkinson und I. Bossenmeier, J. Lab. Clin. Med. 37, 831 (1951). - 28. Schwartz, S. und H. M. Wikoff, J. Biol. Chem. 194, 563 (1952). - 29. Heilmeyer, L., R. Clotten und L. Heilmeyer, Bluthämsynthese Gg. Thieme, Stuttgart (1964). - 30. Rimington, C. und S. L. Svernsson, Scand. J. Clin. u. Lab. Invest. 2, 209 (1950). - 31. Wirh, T. K., Scand. J. Clin. Lab. Invest. 7, 193 (1955). 32. ERIKSEN, L., Scand. J. Clin. Lab. Invest. 10, 319 (1958). - 33. Schwartz, S., M. Glickman, R. Hunter und J. Wallace, Studies of Porphyrin Metabolism III. The Relation of Erythropoiesis to the Excretion of Coproporphyrin by Dogs and Rabbits and to the Concentration of Coproporphyrin and Protoporphyrin in Rabbit Erythrocytes in Zirkle R. E., editor: Biologic effects of External x- and -Radiation, part 2 (August 1956), Atomic Energy Commission, p. 187.

Doz. Dr. R. Clotten 78 Freiburg/Brg. Hugstetter Str. 55

\title{
Urin-Arylamidase
}

\author{
Von W. Jösch und U. C. Dubachi'; \\ Aus der Medizinischen Poliklinik der Universität Basei (Direktor: Prof. Dr. O. Gsell)
}

(Eingegangen am 7. März 1966)

Die Urin-Arylamidase („Leucinaminopeptidase“, „LAP“) wurde mit dem Substrat L-Leucin-p-nitroanilid spektrophotometrisch bei $405 \mathrm{~m} \mu$ bestimmt. - Bei 37 männlichen Erwachsenen im Alter von 20 bis 63 Jahren ergaben sich Grenzwerte von 225-1170 mE im 8-Stdn.-Urin (bzw. 0,6-4,7 mE/ml) bei einem Mittelwert von $602 \pm 262 \mathrm{mE}(2,2 \pm 0,9 \mathrm{mE} / \mathrm{ml})$. Bei 40 weiblichen Erwachsenen im Alter von 18 bis 33 Jahren fanden sich Grenzwerte von 30-798 mE im 8-Stdn.-Urin (bzw. 0,1-3,6 mE/ml) bei einem Mittelwert von $337 \pm 238 \mathrm{mE}(1,3 \pm 0,84 \mathrm{mE} / \mathrm{ml})$.

Der Einfluß der zelligen Elemente des Urins auf die Arylamidase-Aktivität wurde geprüft: obwohl nach Zentrifugieren im zellhaltigen Rückstand mit zunehmender Zahl von Leukocyten und Rundepithelien eine steigende Aktivität gefunden wurde, blieb diejenige des Uberstandes unverändert. - Aspirin steigerte die Ausscheidung von Epithelien aus dem Tubulusapparat um mehr als das Hundertfache. Die Arylamidase-Aktivität des Urins blieb dabei innerhalb der Normgrenzen.

Urinary arylamidase ("leucine aminopeptidase", "LAP") was determined spectrophotometrically at $405 \mathrm{~m} \mu$ with the substrate L-leucinep-nitroanilide. For 37 adult males, aged 20 to 63 years, the values varied between 225 and $1170 \mathrm{~m} \mu$ per $8 \mathrm{hr}$. urine (i. e. $0.6-4.7 \mathrm{mU} / \mathrm{ml}$ ), with an average value of $602 \pm 262 \mathrm{~m} \mu(2.2 \pm 0.9 \mathrm{mU} / \mathrm{ml})$. For 40 adult females, aged 18 to 33 years, the values varied between 30 and $798 \mathrm{~m} \mu$ per $8 \mathrm{hr}$. urine (i. e. $0.1-3.6 \mathrm{mU} / \mathrm{ml}$ ), with an average value of $337 \pm 238 \mathrm{~m} \mu(1.3 \pm 0.84 \mathrm{mU} / \mathrm{ml})$.

The effect of urinary cellular constituents on the arylamidase activity was tested: although the activity in the cellular material sedimented by centrifugation was increased with increasing numbers of leucocytes and renal tubular cells, the activity of the supernatant remained unchanged. Aspirin increased the excretion of epithelia from the uriniferous tubules by more than a hundred fold, but the arylamidase activity of the urine remained within the normal limits.

Als Arylamidase bezeichnen wir ein Enzym, welches die Arylamide L-Leucin $=\beta$-naphthylamid und L-Leucin-pnitroanilid spaltet. Andere Bezeichnungen sind Leucinaminopeptidase („LAP"), „sogenannte“ Leucinaminopeptidase, Aminosäurepeptidase und Aminosäurearylamidase (1-3). - Arylamidase kann regelmäßig im Urin nachgewiesen werden. Den größten Teil dieser normalen Ausscheidung soll das bei der physiologischen Mauserung der Tubulusepithelien aưs den Zellen freigesetzte Enzym darstellen (4). Neuere Untersuchungen über eine klinische Bedeutung der Arylamidase-Bestimmung im Ürin richten sich vor allem auf die Möglichkeit, akute Schädigungen der proximalen Tubuli anhand erhöhter Werte zu erfassen (5-10). Weitgehend

1) Diese Arbeit wurde ermöglicht durch den Schweizerischen Nationalfonds zur Förderung der Wisșenschaftlichen Forschung (Beitrag Nr. 3337 und 3540). ungeklärt sind jedoch noch Fragen der Abhängigkeit der Enzymaktivität von zelligem Sediment, Proteinurie, $\mathrm{pH}$ des Urins, Diuresezustand und möglichen weiteren Faktoren.

Die vorliegende Arbeit beschreibt die Methodik der Arylamidase-Bestimmung im Urin und gibt die mit einer kommerziell erhältlichen Test-Kombination bestimmten Normalwerte an. Daneben wird der Einfluß der zellulären Elemente des Urinsediments einschließlich medikamentös herbeigeführter Steigerung der Ausscheidung von Epithelien der Nierenkanälchen auf die Arylamidase-Aktivität des Urins untersucht.

\section{Methodik}

Nacbweis der Arylamidase

Bis 1964 wurde die Arylamidase im allgemeinen durch die Spaltung von L-Leucin- $\beta$-naphthylamid nachge- 